\title{
MBAC: Impact of the Measurement Error on Key Performance Issues
}

\author{
Anne Nevin, Peder J. Emstad, and Yuming Jiang \\ Centre for Quantifiable Quality of Service in Communication Systems (Q2S) ${ }^{\star}$, \\ Norwegian University of Science and Technology (NTNU), Trondheim, Norway \\ \{anne.nevin, peder.emstad\}@q2s.ntnu.no
}

\begin{abstract}
In Measurement Based Admission Control (MBAC), the decision of accepting or rejecting a new flow is based on measurements of the current traffic situation. Since MBAC relies on measurements, an in-depth understanding of the measurement error and how it is affected by the underlying traffic is vital for the design of a robust MBAC. In this work, we study how the measurement error impacts the admission decision, in terms of false rejections and false acceptances, and the consequence this has for the MBAC performance. A slack in bandwidth must be added to reduce the probability of false acceptance. When determining the size of this slack, the service provider is confronted with the trade-off between maximizing useful traffic and reducing useless traffic. We show how the system can be provisioned to meet a predefined performance criteria.
\end{abstract}

\section{Introduction}

Measurement Based Admission Control (MBAC) has for a long time been recognized as a promising solution for providing statistical Quality of Service(QoS) guarantees in packet switched networks. An MBAC does not require an a priori source characterization which in many cases may be difficult or impossible to attain. Instead, MBAC uses measurements to capture the behavior of existing flows and uses this information together with some coarse knowledge of a new flow, when making an admission decision for this requesting flow. Measurements are unavoidably inaccurate. This imperfection creates uncertainties which affect the MBAC decision process. The degree of uncertainty depends on flow characteristics, the length of the observation window and the flow dynamics. Flows will be accepted when they should have been rejected, false acceptance, and rejected when they should have been accepted, false rejections. For the service provider, false rejections translates into a decrease in utilization and for the end user, false admissions means that the QoS of the flow can no longer be guaranteed. Basing admissions on measurements clearly requires the understanding of the measurement error and how this impacts the performance of MBAC.

\footnotetext{
* "Centre for Quantifiable Quality of Service in Communication Systems, Centre of Excellence" appointed by The Research Council of Norway, funded by the Research Council, NTNU and UNINETT. http://www.q2s.ntnu.no.
} 
Consider a link where the maximum allowable mean rate is $u c$ and the main task is to keep the average workload of the link at or below this level. An MBAC is put in place which uses measurements to find an estimate $\hat{R}$ of the average aggregate rate of accepted flows. When a new flow arrives, with mean rate $\xi$, it will be accepted if:

$$
\hat{R}+\xi \leq u c .
$$

With MBAC, care must be taken, since $\hat{R}$ is not the true mean rate and includes an error, which will cause false admissions and false rejections. The measurements are improved when they are taken over a longer measurement window. However, flows leaving within the window results in flawed estimates, thus the flow lifetimes set an upper limit for the window size. Given this window size, how confident can we be that this is not a false acceptance? Is this good enough? If the answer is no, the reserved bandwidth for the flows $u c$, must be reduced by some slack to make up for the measurement uncertainty. But how large should this slack in bandwidth be?

There is a tradeoff between rejecting too many flows thus wasting resources, and accepting too many flows resulting in QoS violations. In this work we study how the measurement errors and flow dynamics impact the performance of MBAC in terms of proper performances measures. A simple example shows how the system can be provisioned with a predefined performance criteria.

The focus in the literature has been on finding MBAC algorithms that maximize utilization while providing QoS which basically implies the determination of $u c$ (see [1] and 2] for an overview). A deeper analytical understanding of the measurement process and its error has been sought in [3] and [4. Our work differs significantly from previous work, in that we purely focus on the impact inaccurate measurements have on the MBAC decision process. Correlation characteristics within flows are included and we find how the uncertainty in the measurements vary with the length of the observation window. This work is based on previous work [5] and is part of a methodology and design of an analytical framework for analyzing measurement error.

The reminder of the paper is organized as follows. First the system model is introduced in Section 2. Section 3 details the rate level measurements and Section 4 introduces the flow level framework and defines the performance measures. Provisioning is discussed in Section [5] and follows up with a case study in Section 6. before the conclusion is given in Section 7

\section{System Model}

Flows compete for a limited resource, a network link of capacity $c$, controlled by MBAC. The flow's QoS requirement can be guaranteed as long as the average aggregate rate is at or below $u c$, where $u, 0<u<1$ is a tuning parameter. An optimal value for $u$ depends on flow characteristics. In this work, $u$ is assumed a given constant and a discussion around its optimal settings is out of scope. The MBAC works as follows: The MBAC measures the average aggregate rate 
$\hat{R}$ based on observations of the aggregate rate $R(t)$ over a measurement window of size $w$. This measurement replaces the measurement taken in the previous window. When a new flow arrives, it will be accepted or rejected according to (11). Additional flows arriving within the same window will be rejected and lost.

Each flow $i$ is a stationary rate process, $X_{i}(t)$ with mean $\xi$ and covariance $\rho(\tau)$. A mixing of different flow types will cause increased complexity for the MBAC algorithm and also the measurement process. To simplify, only the homogenous case where flows are of the same type, will be considered. With this assumption, the system state $N$ can be specified by the current number of flows. The maximum number of flows the system can handle is thus $n_{\max }=u c / \xi$. It is natural to separate the timescale into the rate level where the measurements are done and the flow level where the admission decision is made. The measurements are taken by means of continuous observation and will be discussed next.

\section{Rate Level: Measurements and Measurement Error}

The flow rate process $X_{i}(t)$ is observed continuously over the window and an estimate of the mean, $\hat{X}_{i}$ is then: [5]:

$$
\hat{X}_{i}=\frac{1}{w} \int_{0}^{w} X_{i}(t) d t
$$

This measured statistic varies with the window size according to [5]:

$$
\zeta^{2}(w)=\frac{2}{w^{2}} \int_{0}^{w}(w-t) \rho(t) d t
$$

The aggregate rate process is also a stationary rate process. Conditioned on being in state $n$, the aggregate mean is $n \xi$. An estimate of the aggregate mean is given by:

$$
\hat{R}=\sum_{i=1}^{n} \frac{1}{w} \int_{0}^{w} X_{i}(t) d t
$$

When the number of flows is in the order of a few tens [6] (e.g 30 flows), the aggregate rate is approximately normally distributed, thus also $\hat{R} \sim \mathcal{N}\left(n \xi, n \zeta^{2}(w)\right)$. This assumption will be made here. The accuracy of this measurement can then be described by the $1-\varepsilon$ confidence interval: $\hat{R}-z_{\frac{\varepsilon}{2}} \sqrt{n} \zeta(w) \leq n \xi<$ $\hat{R}+z_{\frac{\varepsilon}{2}} \sqrt{n} \zeta(w)$, where $z_{\frac{\varepsilon}{2}}$ is the $(1-\alpha / 2)$ quantile of the normal distribution.

It is intuitive to think that in order to achieve a certain measurement accuracy all that is needed is to increase the window size. However in order for the above estimate to hold, the requirement is that no flows leave during the window, i.e. the aggregate rate process is stationary with a known distribution. Otherwise the actual estimate becomes incorrect. The flow lifetime therefore sets an upper limit for the window size. The inaccuracy of the measurement translates into uncertainty in the admission decision process done at the flow level and will be described next. 


\section{Flow Level and Performance Measures}

Let new flows arrive following a Poisson process with parameter $\lambda$. If the flow is accepted it stays in the system for a lifetime that is negative exponentially distributed with mean $1 / \mu$. A flow that is not accepted by the MBAC is lost. The offered flow load is the Erlang load [7] denoted by A. This is the average number of simultaneous flows if there is no blocking given by:

$$
A=\lambda \cdot E\left(T_{L}\right) .
$$

With the assumption that $\hat{R} \sim \mathcal{N}\left(i \xi, i \zeta^{2}(w)\right)$, if there are $N=i$ flows in the system, a new arriving flow will be accepted with a probability $q_{i}=P(\hat{R}+\xi \leq$ $\left.n_{\max } \mid N=i\right)$. We will assume that the arrival rate is such that the probability of more than one flow arrival per window is very small. The lost traffic due to multiple arrivals within the window is thus very small and can be neglected. A flow upon arrival is promptly admitted or rejected, which justifies modeling the admission process as a loss system.

The number of flows currently accepted by the MBAC then follows a continuous time Markov chain, see Fig. 1 and the probability that there are $i$ flows in the system is:

$$
P(i)=\frac{\frac{A^{i}}{i !} \prod_{x=0}^{i-1} q_{x}}{\sum_{j=0}^{\infty} \frac{A^{j}}{j !} \prod_{x=0}^{j-1} q_{x}}
$$

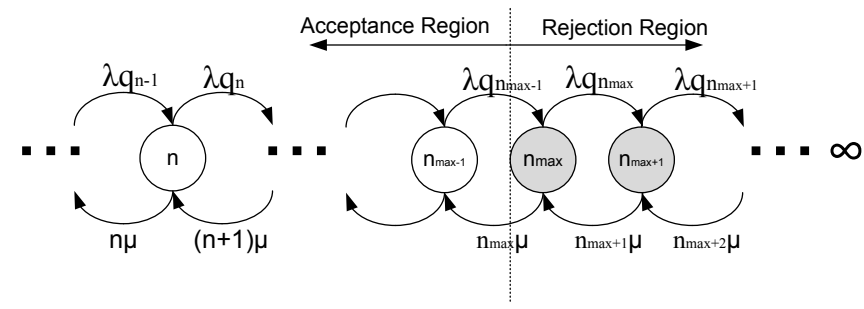

Fig. 1. State diagram of the number of sources accepted by the MBAC

In [8] a similar framework is defined, where the $q_{i} s$ are based on the probability that the instantaneous rate measurements are above a threshold. However, they do not specify how these measurements should be taken. Since the instantaneous rate is a random quantity without a true value, it can not be measured [9]. This is where our work deviates significantly from [8]. We study the measurement error in isolation and the $q_{i} s$ depend on how accurate the measurements are.

Implied by (6) and as also discussed in [8, the distribution $P(i)$ is indeed insensitive to the distribution of the flow lifetime and only depends on the expected flow lifetime. 
The system state space can be divided into two regions; the acceptance region, $N<n_{\max }$ and the rejection region, $N \geq n_{\max }$ see Fig. 1.

Rejecting a flow when the system is in the acceptance region is a False rejection and accepting a flow when the system is in the rejection region is a False acceptance.

For assessing the performance and provisioning purposes, we define the following flow level performance measures:

- Probability of False acceptance, $P_{F A c c}$, is the probability that an arriving flow is accepted in the rejection region

$$
P_{F A c c}=\sum_{i=n_{\max }}^{\infty} P(i) \cdot q_{i}
$$

- Probability of False rejection, $P_{F R e j}$, is the probability that an arriving flow is rejected in the acceptance region

$$
P_{F R e j}=\sum_{i=1}^{n_{\max }-1}\left(1-q_{i}\right) P(i)
$$

- Blocking probability, $P_{B}$, is the probability that an arriving flow is rejected.

$$
P_{B}=P_{F R e j}+P\left(N \geq n_{\text {max }} \cap \text { rejection }\right)=\sum_{i=1}^{\infty}\left(1-q_{i}\right) P(i)
$$

- Carried useful traffic, $A_{\text {useful }}$, is the expected number of flows in the acceptance region.

$$
A_{\text {useful }}=\sum_{i=0}^{n_{\max }} i P(i)
$$

- Carried useless traffic, $A_{\text {useless }}$, is the expected number of flows in the rejection region.

$$
A_{\text {useless }}=\sum_{i=n_{\max }+1}^{\infty} i P(i)
$$

- Lost Traffic, $A_{\text {lost }}$, is the traffic that is blocked from the network

$$
A_{\text {lost }}=A P_{B}
$$

If there are no measurement errors, the admission controller becomes ideal, $\hat{R}=\bar{R}$ and the distribution of flows is then as for the Erlang Loss system. This system only carries useful traffic and arriving flows will experience a blocking probability given by the Erlang B formula. 


\subsection{Flow Load and Window Size Limitations}

In the analytical formulas used for the performance evaluation, when increasing $A$, it is indifferent if this is done by increasing the mean flow lifetime $1 / \mu$ or increasing the arrival rate $\lambda$. In reality this is not true. Many arrivals within a measurement window will increase the blocking probability since the MBAC only admits at most one flow after a measurement update. When predicting the performance using the analytical formulas, we accept just one arrival within a window. It is assumed that the blocking probability due to multiple arrivals is negligible.

For the accepting probabilities, the assumption is that the number of flows is constant during a measurement window. If flows leave during the window, then the theoretically predicted performance will be optimistic compared to actual performance in terms of system utilization. Given a constant $A$, a longer measurement window (thereby reducing the measurement error) can be used for long lifetimes (infrequent arrivals) as compared to short lifetimes (frequent arrivals).

The performance measures can be directly stated upfront using the defined formulas if: 1) The lost traffic due to previous arrivals within the window can be ignored 2) The probability of a flow leaving within a measurement window is small for the actual parameter values $\mu$ and $n 3$ ) In addition we assume that the correlation at arrival points can be neglected.

\section{$5 \quad$ Provisioning}

The QoS provided to the flows can only be guaranteed as long as the number of flows is at or below $n_{\max }$, thus admitting more than $n_{\max }$ flows should be avoided. If the probability of false acceptance is too high, a safeguard in terms of a slack in bandwidth can be added to make up for the measurement errors.

As in Paper [5], let the safeguard have increments of size size $l \xi, 0<l<n_{\max }$ and the refined admission control algorithm becomes:

$$
\hat{R}+\xi \leq \xi n_{\max }-l \xi
$$

The critical situation arises as soon as the system reaches state $n_{\max }$, where accepting a flow will result in the first false admission. With the condition that the system is in state $n_{\max }$ we define the conditional performance requirement:

$$
P\left(F A c c \mid N=n_{\max }\right)=P\left(\hat{R}+\xi \leq \xi n_{\max } \mid N=n_{\max }\right) \leq \epsilon
$$

where $\epsilon$ is termed the conditional performance target.

For a given quantile and predefined window size, $P\left(F A c c \mid N=n_{\max }\right)$ can be kept below the target if the number of levels is [5]:

$$
l+1=\left\lceil\frac{\sqrt{n_{\max }} \zeta(w) z_{\varepsilon}}{\xi}\right\rceil .
$$


Where $z_{\varepsilon}$ is the $\varepsilon$ - quantile of the standard normal distribution The resulting $l$ can be used for provisioning the system.

Since the MBAC solely estimates the number of flows through measurements, $l$ is independent of the system state and when there are $i$ flows in the system, a new arriving flow will be accepted by the MBAC, with a probability $q_{i}=$ $P\left(\hat{R}+\xi \leq n_{\max }-l \mid N=i\right)$.

The size of $l$ controls the probability of entering the rejection region by forcing the probability distribution towards left (Fig. 1). Obviously, if the slack is too large, the MBAC becomes too pessimistic and resources are wasted unnecessarily. The actual performance can be evaluated by means of the performance measures defined in Section 4. To the customer, the performance measures of interest are the blocking probability and the probability of false acceptance. The service provider seeks to balance the carried useless traffic and carried useful traffic.

For a given flow load the network can be provisioned to meet a desired performance target. But what flow load should be used?

The network should be dimensioned to ensure a small blocking probability under normal loads, say $P_{B}<0.01$. At such low loads the probability of entering the rejection region is very small and excellent QoS can be provided to all flows. The problem arises in times of excessive demand. With an ideal controller, when the load increases above what is predicted the blocking probability increases to unacceptable high values. However, the QoS to the already admitted flows will not be harmed. With MBAC on the other hand, also the probability of false acceptance and useless traffic increase with increasing loads. Reviewing work in the MBAC literature, it is common practise to test the MBAC performance under a heavy flow load. For example a load corresponding to $50 \%$ blocking probability is used in [1] and an infinite load is used in [3].

We do not attempt to answer, exactly what load to use for provisioning purposes. The load must be relatively high, since the main task of MBAC is to preserve QoS to its users when the load exceeds normal values [10. Obviously at such loads, the normal blocking probability ( e.g $P_{B}=0.01$ ) can not be met.

\section{Case Study Using MMRP Source Models}

In this section provisioning to fulfill some predefined performance criteria will be demonstrated with an example.

Let the flows be modeled by a two-state Markov modulated rate process (MMRP) which is a simple, yet realistic source model used to model both speech sources and video sources [11]. The MMRP process, $X(t)=r I(t)$ where $I(t)$, alternates between states $I=0$ and $I=1$ where $r$ is the peak rate. The duration of the 0 and 1 states follows a negative exponential distribution with mean $1 / \alpha$ and $1 / \beta$ respectively.

The variance of the time average of such a source is [5]:

$$
\zeta^{2}(w)=\frac{2 r^{2} \alpha \beta}{w^{2}(\alpha+\beta)^{3}}\left(w-\frac{1}{\alpha+\beta}\left(1-e^{-w(\alpha+\beta)}\right)\right)
$$




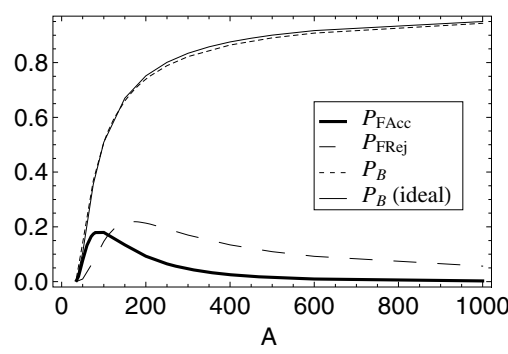

(a) $P_{F R e j}$ and $P_{F A c c}$ vs load

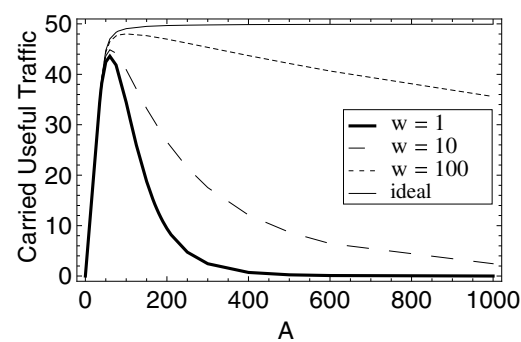

(b) Carried useful traffic

Fig. 2. Probability of false acceptance as the load increase and the corresponding Carried Useful Traffic

In the following, $\alpha=\beta=r=2$, and $\xi$ is then 1 . The flows have a QoS requirement that can only be guaranteed as long as $N \leq n_{\max }=50$.

First we shall show how the performance measures are impacted when the offered flow load, $A$ is varied. At this stage we use no slack in the bandwidth (e.i. $l=0)$ Keeping the window size constant at $w=1$, Fig. 2(a) shows how the performance measures $P_{F A c c}, P_{F R e j}$ and $P_{B}$ are affected when the flow load increases. Low loads result in negligible false acceptance. Instead false rejections cause a slight increase in blocking probability as compared to the ideal. At a load of about $A=60, P_{F A c c}=P_{F r e j}$ and then as the load increases $P_{F A c c}$ increases resulting in a slightly lower blocking probability as compared to the $i d e a l$. As the load increases towards infinity, $P_{F A c c}$ becomes zero. The reason for this is that the system moves into the rejection region and will eventually only carry useless traffic. This is illustrated in Fig. 2(b) which shows that as the load increases the carried useful traffic approaches zero. Also shown, is that for larger window sizes, the MBAC approaches the ideal and the carried useful traffic falls off slower.

We are interested in balancing the tradeoff between the overall probability of false rejection and probability of false acceptance. For the user, the main concern is the probability of false acceptance and in this example the requirement is $P_{F A c c}<0.01$. Let the offered flow load be $A=100$ and let the window size be $w=1$. The performance plots shown in Fig. 3(a) 3(b) 3(c), and 3(d) illustrate the tradeoff between blocking and accepting flows when $P\left(F A c c \mid N=n_{\max }\right)$ is varied. Consider first the performance in the light of the customer. To fulfill the requirement of $P_{F A c c}<0.01$, Fig $3(\mathrm{a})$, shows that $P\left(F A c c \mid N=n_{\max }\right)<0.17$. At this value the blocking probability is about $5 \%$ larger than for the ideal.

For the service provider, the concern is false rejections and useless traffic. As $P\left(F A c c \mid N=n_{\max }\right)$ increases, false rejections fall off ( see Fig. 3(b)) and the useless traffic increases (see Fig 3(c) . Observe in Fig $3(\mathrm{~d})$ that a value $P\left(F A c c \mid N=n_{\max }\right)=0.15$ maximizes the carried useful traffic. Reducing the value and the admission controller becomes too strict due to the increase in $P_{F R e j}$. Increasing the value passed this point on the other hand and the 
admission controller accepts too much useless traffic. In this case, using $P(F A c c \mid$ $\left.N=n_{\max }\right)=0.15$, also ensures that $P_{F A c c}<0.01$.

This is an interesting fact since it shows that the carried useful traffic can be maximized by correctly tuning $P\left(F A c c \mid N=n_{\max }\right)$. This value of $P(F A c c \mid$ $\left.N=n_{\max }\right)$ can be directly mapped to a safeguard in terms of levels (15). The carried useful traffic can thus be increased by adding a safeguard. However if the safeguard is too large, the carried useful traffic will decrease because the MBAC becomes too strict.

The value of $l$ which maximizes the carried useful traffic, will depend on the window size and the offered flow load. With a higher flow load $A$, the value of $l$ which maximizes the carried useful traffic will increase. For example, the extreme load of $A=1000$ results in $l=9$. Decreasing the load, will have the opposite effect, and eventually as the load is reduced further adding a safeguard will only decrease utilization.

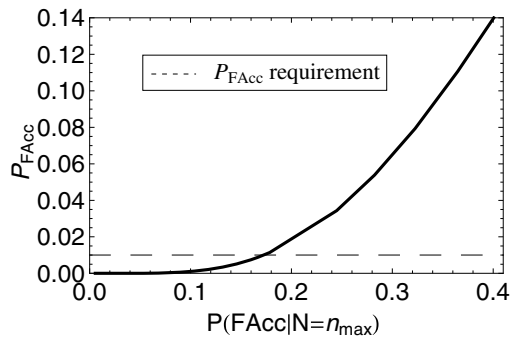

(a) $P_{F A c c}$

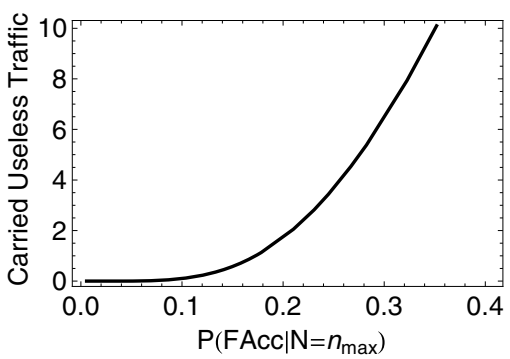

(c) Carried useless traffic

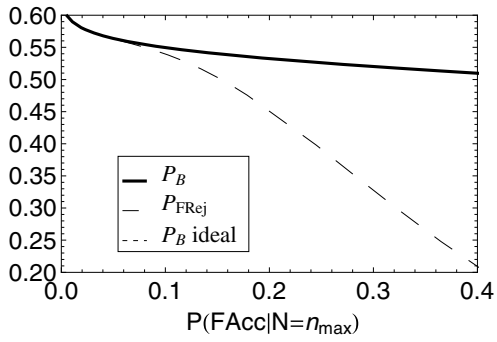

(b) $P_{B}$ and $P_{F R e j}$

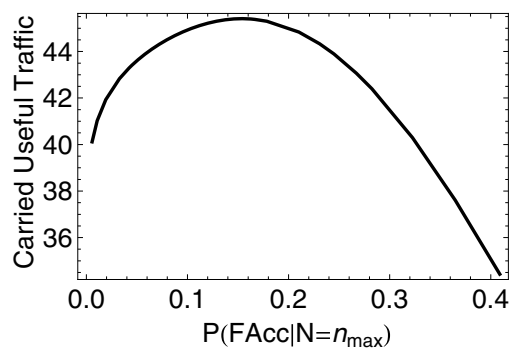

(d) Carried useful traffic

Fig. 3. The performance measures as $P\left(F A c c \mid N=n_{\max }\right)$ varies for $A=100$ and $w=10$ a)Probability of false acceptance, b) Probability of false rejection and overall blocking probability, c) Carried useless traffic and d) Carried useful traffic

Numerous simulations (with MMRP sources) to check the analytical formulas are omitted due to limited space. As long as there is no correlation between measurement windows, these simulations indicated that the formulaes are valid even when the number of aggregated flows is as low as 3 . 


\section{Conclusion}

This current work gives an in-depth understanding of how measurement uncertainties and flow dynamics impact the MBAC admission decision. An MBAC algorithm, no matter how advanced, is of little use if the measurement errors are not taken into account. These errors translate into uncertainty in the decision process. The degree of uncertainty abates with the length of the observation window. Despite the heavy reliance on measurements, there is in the literature of MBAC, surprisingly very limited work focusing on the impact of the measurement error and how it affects the admission decision.

The probability of false admissions can be reduced by adding a slack in bandwidth. However, if the slack is too large, flows are blocked unnecessarily. With some appropriate performance measures, we showed how the system can be provisioned to meet a predefined performance criteria.

In this work, we assumed that the flows were homogenous, however in a separate work we have developed the analytical tools needed to extend this work to the non-homogenous case.

\section{References}

1. Breslau, L., Jamin, S., Shenker, S.: Comments on the performance of measurementbased admission control algorithms. In: IEEE INFOCOM (2000)

2. Moore, A.W.: Measurement-based management of network resources. Technical Report, University of Cambridge, Cambridge CB3 OFD, United Kingdom (April 2002)

3. Grossglauser, M., Tse, D.N.: A time-scale decomposition approach to measurementbased admission control. IEEE/ACM Trans. Networking 11(4), 550-563 (2003)

4. Dziong, Z., Juda, M., Mason, L.G.: A framework for bandwidth management in ATM networks - aggregate equivalent bandwidth estimation approach. IEEE/ACM Trans. Networking 5(1), 134-147 (1997)

5. Nevin, A., Emstad, P., Jiang, Y., Hu, G.: Quantifying the uncertainty in measurements for mbac. In: Oliver, M., Sallent, S. (eds.) EUNICE 2009. LNCS, vol. 5733, pp. 1-10. Springer, Heidelberg (2009)

6. van de Meent, R., Mandjes, M., Pras, A.: Gaussian traffic everywhere? In: IEEE International Conference on Communications, ICC 2006, June 2006, vol. 2, pp. 573-578 (2006)

7. Iversen, V.B.: Teletraffic engineering and network planning, course Textbook (May 2006)

8. Gibbens, P.K.R.J., Kelly, F.P.: A decision-theoretic approach to call admission control in atm networks. IEEE Journal on Selected Areas in Communications (JSAC) 13(6), 1101-1114 (1995)

9. Rabinovich, S.G.: Measurement Errors and Uncertaintees, 2nd edn. Springer, New York (2005)

10. Roberts, J.W.: Internet traffic, qos and prising. Proceedings of the IEEE 92(9) (2004)

11. Schwartz, M.: Broadband integrated networks. In: Becker, P. (ed.), Prentice Hall, Englewood Cliffs (1996) 